\title{
As equipes multidisciplinares como dispositivos "técnicos de referência" em saúde mental nos caps e a gestão do cuidado: uma revisão integrativa de literatura
}

\author{
Multidisciplinary teams as "technical reference" devices in mental health in caps and care \\ management: an integrative literature review \\ Equipos multidisciplinarios como dispositivos de "referencia técnica" en salud mental en gapas y \\ gestión de cuidados: una revisión integrativa de la literatura
}

Recebido: 04/05/2021 | Revisado: 10/05/2021 | Aceito: 11/05/2021 | Publicado: 28/05/2021

\author{
Patrícia Socorro Coelho Portal \\ ORCID: https://orcid.org/0000-0002-0080-6675 \\ Fundação Santa Casa de Misericórdia, Brasil \\ E-mail: patriciaportal104@gmail.com \\ Thaís de Oliveira Carvalho Granado Santos \\ ORCID: https://orcid.org/0000-0001-9496-4561 \\ Fundação Santa Casa de Misericórdia, Brasil \\ E-mail: thaisgranadosantos@gmail.com \\ Simony do Socorro de Vilhena Guimarães \\ ORCID: https://orcid.org/0000-0002-3607-7929 \\ Fundação Santa Casa de Misericórdia, Brasil \\ E-mail: simony.pa@gmail.com \\ Michele de Pinho Barreiros \\ ORCID: https://orcid.org/0000-0002-7444-6238 \\ Fundação Santa Casa de Misericórdia, Brasil \\ E-mail: michelepinhobarreiros@gmail.com \\ Rejane Brandão Pinto \\ ORCID: https://orcid.org/0000-0002-0416-0664 \\ Fundação Santa Casa de Misericórdia, Brasil \\ E-mail: rejane23@msn.com \\ Cláudia Heidtmann Dias \\ ORCID: https://orcid.org/0000-0002-4118-8551 \\ Fundação Santa Casa de Misericórdia, Brasil \\ E-mail: clauheidtmann@gmail.com \\ Pilar Maria de Oliveira Moraes \\ ORCID: https://orcid.org/0000-0003-2817-4574 \\ Fundação Santa Casa de Misericórdia, Brasil \\ E-mail: pilarmoraesnutri@gmail.com \\ Xaene Maria Fernandes Duarte Mendonça \\ ORCID: https://orcid.org/0000-0002-0958-276X \\ Fundação Santa Casa de Misericórdia, Brasil \\ E-mail: xaene@ufpa.br
}

\begin{abstract}
Resumo
Objetivamos realizar uma revisão integrativa da literatura acerca da gestão do cuidado e as equipes multidisciplinares em saúde mental estudos publicados em periódicos entre os anos de 2015 e 2020. Metodologicamente foi utilizado uma revisão integrativa da literatura estabelecendo uma pergunta norteadora: a equipe multidisciplinar como dispositivos "técnicos de referência" em saúde mental nos CAPS possui capacitação para a gestão do cuidado em saúde mental tal como possuem conhecimento acerca de suas atribuições? Definiu-se as bases de dados: LILACS, SciELO, PubMed Central e MEDLINE e descritores combinados com operadores booleanos: "CAPS" e "gestão do cuidado" e "técnicos de referência" e "equipe multidisciplinar" e "saúde mental" e "Reference technicians" e "Multidisciplinary team" e "Mental health" e "Care management". Logo após, estabelecemos os critérios de inclusão exclusão; categorização dos estudos e extração dos dados; avaliação dos estudos incluídos na revisão; discussão e interpretação dos resultados e a síntese das informações evidenciadas nos artigos. Como resultado, foram revisados 147 artigos científicos e apenas 15 fizeram parte desse estudo definindo assim, duas subcategorias para análise dos artigos: aspectos desfavoráveis e desfavoráveis em relação à gestão do cuidado e equipe multidisciplinar como dispositivos em saúde mental em CAPS. Concluímos que é de suma importância uma interação singular e o envolvimento de todos os membros da equipe multidisciplinar como dispositivos técnicos de referência nos CAPS a adoção de medidas mais eficazes. $O$ trabalho em equipe pode se tornar diferenciado ao ponto de olhar para o paciente como um todo e proporcionar um atendimento mais humanizado.
\end{abstract}


Palavras-chave: Assistência à saúde mental; Saúde mental; Centros de atenção psicossocial; Serviços comunitários de saúde mental.

\begin{abstract}
We aim to carry out an integrative literature review about care management and multidisciplinary teams in mental health studies published in journals between the years 2015 and 2020. Methodologically, an integrative literature review was used, establishing a guiding question: the multidisciplinary team as devices " reference technicians "in mental health at CAPS have training for the management of mental health care as they have knowledge about their duties? The databases were defined: LILACS, SciELO, PubMed Central and MEDLINE and descriptors combined with Boolean operators: CAPS" or "gestão do cuidado" and "Técnicos de referência" and "equipe multidisciplinar" and "saúde mental" and "Reference technicians" and "Multidisciplinary team" and "Mental health" and "Care management". After that, we established the inclusion exclusion criteria; categorization of studies and data extraction; evaluation of studies included in the review; discussion and interpretation of results and synthesis of the information evidenced in the articles. As a result, 147 scientific articles were reviewed and only 15 were part of this study, thus defining two subcategories for analyzing the articles: unfavorable and unfavorable aspects in relation to the management of care and the multidisciplinary team as mental health devices in CAPS. We conclude that a unique interaction and the involvement of all members of the multidisciplinary team as technical devices of reference in the CAPS is of paramount importance in adopting more effective measures. Teamwork can become differentiated to the point of looking at the patient as a whole and providing more humanized care.
\end{abstract}

Keywords: Mental health assistance; Mental health; Mental health services; Community mental health services.

\title{
Resumen
}

Nuestro objetivo es realizar una revisión integradora de la literatura sobre la gestión del cuidado y los equipos multidisciplinarios en los estudios de salud mental publicados en revistas entre los años 2015 y 2020. Metodológicamente, se utilizó una revisión integradora de la literatura, estableciendo una pregunta orientadora: ¿el equipo multidisciplinario como dispositivos "referencia los técnicos" en salud mental del CAPS tienen formación para la gestión de la atención en salud mental ya que tienen conocimiento sobre sus funciones? Se definieron las bases de datos: LILACS, SciELO, PubMed Central y MEDLINE y descriptores combinados con operadores booleanos: CAPS" or "gestão do cuidado" and "Técnicos de referência" and "equipe multidisciplinar" and "saúde mental" and "Reference technicians" and "Multidisciplinary team" and "Mental health" and "Care management". Poco después, establecimos los criterios de exclusión de inclusión; categorización de estudios y extracción de datos; evaluación de estudios incluidos en la revisión; discusión e interpretación de resultados y síntesis de la información evidenciada en los artículos. Como resultado, se revisaron 147 artículos científicos y solo 15 formaron parte de este estudio, definiendo así dos subcategorías para el análisis de los artículos: aspectos desfavorables y desfavorables en relación a la gestión del cuidado y el equipo multidisciplinario como dispositivos de salud mental en CAPS. Concluimos que una interacción única y la implicación de todos los miembros del equipo multidisciplinar como dispositivos técnicos de referencia en los CAPS es de suma importancia para adoptar medidas más eficaces. El trabajo en equipo puede diferenciarse hasta el punto de mirar al paciente como un todo y brindar una atención más humanizada.

Palabras clave: Atención a la salud mental; Salud mental; Servicios de salud mental; Servicios comunitarios de salud mental.

\section{Introdução}

A Reforma Psiquiátrica no Brasil nasce durante a década de 1980, assim como o primeiro Centro de Atenção Psicossocial, intitulado CAPS Professor Luís da Rocha Cerqueira (Filho \& Souza, 2017). Os CAPS são considerados referências na Rede de Atenção Psicossocial (RAPS) de acordo com a Portaria n. 336/2002 (Brasil, 2002), os quais tem caráter aberto, comunitário e territorializado, sendo composto por equipe multiprofissional atuante sob a ótica interdisciplinar e realizando o atendimento às pessoas com sofrimento ou transtorno mental, incluindo aquelas com necessidades decorrentes do uso prolongado de álcool e outras drogas; assim, configuram-se como substitutivos ao modelo asilar.

Em consonância, os profissionais de diversas áreas que atuam nos CAPS compõem as equipes multidisciplinares, sendo que profissionais de nível superior, como enfermeiros, psicólogos, médicos (psiquiatras), assistentes sociais, terapeutas ocupacionais, entre outros, atuam como dispositivos "técnicos de referências" em saúde mental; entretanto cabe destacar a importância dos demais componentes: administrador, técnico de enfermagem, técnico administrativo e auxiliar de serviços gerais, que fazem parte da equipe de cuidado em saúde mental. Assim, o técnico de referência é definido como aquele que tem 
como responsabilidade o monitoramento do usuário, o projeto terapêutico individual, o contato com a família e a avaliação de metas traçadas no projeto (Brasil, 2004).

Apesar do crescimento do interesse de pesquisadores em Saúde Mental, relacionadas ao processo da reforma psiquiátrica e serviços, observa-se que o interesse pela pesquisa sobre a gestão do cuidado em saúde psicológica e o conhecimento produzido na área não vem sendo tão acessível ao cotidiano dos dispositivos "técnicos de referência" em saúde mental nos CAPS (Filho \& Souza, 2017).

Em conformidade, cabe destacar o distanciamento entre o conhecimento produzido em relação à gestão do cuidado e o trabalho proposto pelas equipes multidisciplinares como dispositivos "técnicos de referência" em saúde mental. Uma possível explicação para este distanciamento decorre de que os CAPS não fazem uma preparação para os profissionais atuantes, assim, se estende para mais um obstáculo em que muitos destes profissionais de referência em saúde mental apesar de terem formação, aparentam não possuírem clareza da sua função; podendo influenciar o pensamento e o agir profissional em relação ao gerenciamento do cuidado. Dessa forma, o que se visualiza é que nem todos os profissionais atuantes dentro dos CAPS têm acesso à capacitação ou entendimento conceitual da função de técnico de referência. Contudo, sabemos que o dispositivo "técnicos de referência" é uma forma de fazer a organização dos equipamentos substitutivos em saúde mental (Filho \& Souza, 2017).

Para tanto, é fundamental que a equipe multidisciplinar atuante como técnicos de referência se apropriem do processo de cuidar, não apenas o desenvolvimento de tarefas limitadas, burocráticas e rotineiras, mas é imprescindível estar engajado no contexto enquanto profissional e ser humano, político e social, fazendo assim, a mediação entre a profissão, bem como a organização no cumprimento das normas, com os interesses pessoais e dos clientes nessa questão do cuidar, além de desconstruir o pensamento de uma cultura manicomial dentro de um CAPS, no contexto de seu trabalho (Filho \& Souza, 2017).

Neste contexto, os métodos meta-científicos são apontados como uma maneira eficaz para avaliação do conjunto da produção científica em uma determinada área do conhecimento. Tal tipo de avaliação é feita a partir da revisão de informações em fontes de publicação científica, bases de indexação de dados, bancos de teses e dissertações, periódicos científicos, gerando indicadores importantes da produção, sintetizando as principais ideias, hipóteses, métodos, resultados, bem como o impacto da produção. Nesse sentido, a revisão integrativa permite a busca, a avaliação crítica e a síntese das evidências disponíveis do tema investigado (Ganong, 1987; Whittemore \& Knaf, 2005).

O presente estudo foi delineado com o objetivo realizar uma revisão integrativa da literatura acerca da relação entre gestão do cuidado e equipe multidisciplinares entre estudos publicados em periódicos.

\section{Metodologia}

Trata-se de uma revisão integrativa da literatura, definida como um instrumento de obtenção, identificação, análise e síntese das publicações referentes a um tema específico, permitindo construir uma análise ampla da literatura, abordando inclusive discussões sobre métodos e resultados.

Segundo Mendes et al (2008) a revisão integrativa compreende as seguintes etapas: Identificação da questão norteadora, estabelecimento dos critérios de inclusão e exclusão dos artigos, busca nas bases de dados para identificação dos estudos; Categorização dos estudos e extração dos dados; Avaliação dos estudos incluídos na revisão; Discussão e interpretação dos resultados; Síntese das informações evidenciadas nos artigos. A questão norteadora definida para este estudo foi: A equipe multidisciplinar como dispositivos "técnicos de referência" em saúde mental nos CAPS possui capacitação para a gestão do cuidado em saúde mental tal como possuem conhecimento acerca de suas atribuições? 
Neste contexto, os métodos meta-científicos são apontados como uma maneira eficaz para avaliação do conjunto da produção científica em uma determinada área do conhecimento. Tal tipo de avaliação é feita a partir da revisão de informações em fontes de publicação científica, bases de indexação de dados, bancos de teses e dissertações, periódicos científicos, gerando indicadores importantes da produção, sintetizando as principais ideias, hipóteses, métodos, resultados, bem como o impacto da produção. Nesse sentido, a revisão integrativa permite a busca, a avaliação crítica e a síntese das evidências disponíveis do tema investigado (Ganong, 1987; Whittemore \& Knaf, 2005).

Durante o período de Agosto a Outubro de 2020, foram incluídos na revisão artigos completos relacionados ao objeto de pesquisa, sem restrição de idiomas, originados de periódicos nacionais e internacionais, indexados nas bases de dados referidas, no período 2015 a 2020 (5 anos). Foram excluídos artigos do tipo comentário.

A seleção da amostra deu-se por meio do acesso às bases de dados: Literatura Latino-Americana e do Caribe em Ciências da Saúde (LILACS), Scientific Electronic Library Online (SciELO), US National Library of Medicine (PubMed Central) e Medical Literature Analysis and Retrieval System Online (MEDLINE). Foram usados os descritores controlados a partir da associação dos Descritores em Ciências da Saúde (DeCS) e Medical Subject Headings (MeSH) combinados com operadores booleanos: "CAPS" or "gestão do cuidado" and "Técnicos de referência" and "equipe multidisciplinar" and "saúde mental" and "Reference technicians" and "Multidisciplinary team" and "Mental health" and "Care management"

Visando à sistematização dos dados, desenvolveu-se um instrumento de coleta contendo: autores, ano, bases de dados, periódico, país, características metodológicas dos artigos e resultados alcançados. Após coleta dos dados, procedeu-se à caracterização do corpus de análise.

O registro das informações obtidas nos artigos selecionados deu-se em um instrumento estrutural, conforme Figura 1. O qual foi construído após a leitura dos artigos na íntegra, tendo em vista a questão norteadora do estudo. Foram identificados 720 artigos nas bases de dados LILACS, SciELO, MEDLINE e PubMed. Destes, 583 artigos foram excluídos já que estavam fora do eixo temático, restando 147 artigos para serem submetidos aos critérios de inclusão e exclusão. Primeiramente foram excluídos 77 artigos que não estavam disponíveis de forma online em texto completo. Após esta fase, foram excluídos os estudos que estavam fora do período em questão. Desta forma, restaram 54 artigos. Procedeu-se a leitura dos resumos desses 54 artigos, sendo excluídos 39, pois não respondiam à questão norteadora (18 eram artigos de reflexão, 06 de ensino, 06 sobre reforma psiquiátrica, 05 relatos de experiência e 04 sobre psiquiatria forense).

Ao final, restaram então 15 (quinze) artigos para serem lidos na íntegra. Em relação aos aspectos éticos, foram respeitadas as ideias dos autores, suas definições e conceitos apresentados nos artigos analisados 
Figura 1. Fluxograma da coleta dos artigos nas bases de dados no período de 2015 a 2020.

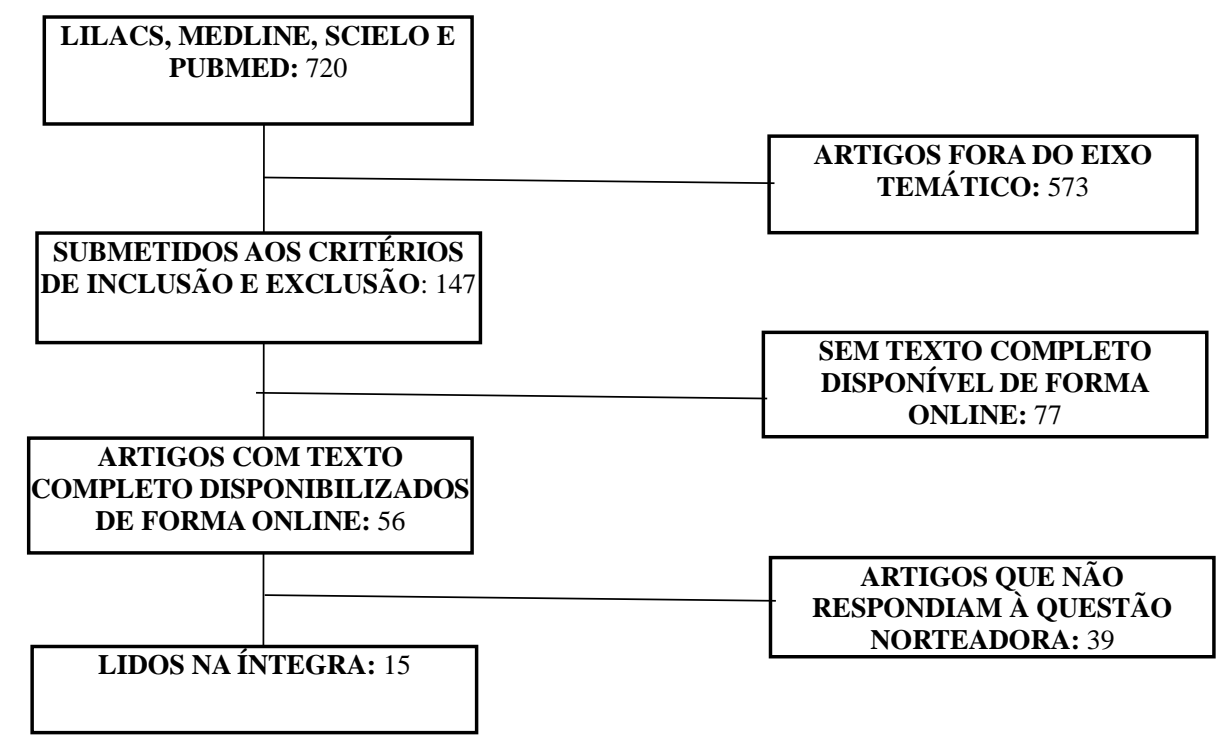

Fonte: Autores (2021).

\section{Resultados}

A partir da análise dos 15 artigos selecionados, apresenta-se no Quadro 1 os resultados quanto ao título do artigo, nomes dos autores, ano e o periódico da publicação. Em relação à profissão dos autores dos artigos da amostra, 32 (66,66\%) dos participantes foram enfermeiros, $11(22,91 \%)$ psicólogos e $05(10,41 \%)$ são médicos, totalizando 48 profissionais. Dos artigos do tipo "empírico" selecionados para o estudo, a maioria foi produzida na região nordeste, totalizando 07 produções científicas, seguida pelas regiões sudeste, com 04 estudos, e a região sul com 03 estudos. Totalizando 14 produções empíricas além de 01 revisão bibliográfica. 
Quadro 1. Distribuição das referências incluídas na revisão integrativa, de acordo com os títulos, autores, ano de publicação e periódicos.

\begin{tabular}{|c|c|c|c|c|}
\hline $\mathbf{N}^{\mathbf{0}}$ & Título & Autores & Ano & Revista \\
\hline 1 & $\begin{array}{l}\text { Qualidade do cuidado em dois centros de } \\
\text { atenção psicossocial sob o olhar de usuários }\end{array}$ & Andrade \& Bosi & 2015 & Saúde e Sociedade \\
\hline 2 & $\begin{array}{c}\text { Processos de trabalho dos profissionais dos Centros de Atenção } \\
\text { Psicossocial: revisão integrativa }\end{array}$ & Pinho et al. & 2015 & $\begin{array}{l}\text { Ciência e Saúde } \\
\text { Coletiva }\end{array}$ \\
\hline 3 & $\begin{array}{l}\text { O apoio matricial em saúde mental: } \\
\text { uma ferramenta apoiadora da atenção à crise }\end{array}$ & $\begin{array}{c}\text { Lima \& } \\
\text { Dimenstein }\end{array}$ & 2016 & $\begin{array}{l}\text { Comunicação, } \\
\text { Saúde e Educação }\end{array}$ \\
\hline 4 & $\begin{array}{l}\text { Apoio Matricial e CAPS: desafios do cenário } \\
\text { na implantação do matriciamento em saúde } \\
\text { mental }\end{array}$ & Salvador \& Pio & 2016 & Saúde em Debate \\
\hline 5 & $\begin{array}{c}\text { A equipe enquanto lugar de formação: } \\
\text { a educação permanente em um Centro de Atenção Psicossocial } \\
\text { álcool e outras drogas }\end{array}$ & $\begin{array}{l}\text { Silva \& } \\
\text { Knobloch }\end{array}$ & 2016 & $\begin{array}{l}\text { Comunicação, } \\
\text { Saúde e Educação }\end{array}$ \\
\hline 6 & $\begin{array}{l}\text { O papel da atenção primária de saúde na constituição das redes } \\
\text { de cuidado em saúde mental }\end{array}$ & Barbosa et al. & 2017 & $\begin{array}{l}\text { Revista de } \\
\text { pesquisa: cuidado é } \\
\text { fundamental online }\end{array}$ \\
\hline 7 & $\begin{array}{c}\text { A percepção sobre o trabalho em equipe } \\
\text { multiprofissional dos trabalhadores de um Centro de Atenção } \\
\text { Psicossocial em Salvador, Bahia, Brasil }\end{array}$ & Filho \& Souza & 2017 & $\begin{array}{l}\text { Comunicação, } \\
\text { Saúde e Educação }\end{array}$ \\
\hline 8 & O Cuidado nos CAPS numa Região de Saúde Maranhense & Borges et al. & 2018 & Rev. Polis e Psique \\
\hline 9 & $\begin{array}{l}\text { O trabalho multiprofissional nos } \\
\text { Centros de Atenção Psicossocial de São Paulo }\end{array}$ & $\begin{array}{l}\text { Jafelice \& } \\
\text { Marcolan }\end{array}$ & 2018 & $\begin{array}{l}\text { Revista Brasileira } \\
\text { de Enfermagem }\end{array}$ \\
\hline 10 & $\begin{array}{l}\text { Gestão do cuidado e interdisciplinaridade: desafios do cotidiano da } \\
\text { atenção psicossocial }\end{array}$ & Pessoa et al. & 2018 & Rev. Salud Pública \\
\hline 11 & $\begin{array}{l}\text { Gestão do cuidado na saúde mental sob a perspectiva da rede de } \\
\text { atenção à saúde }\end{array}$ & Sarzana et al. & 2018 & $\begin{array}{c}\text { Mineira de } \\
\text { Enfermagem }\end{array}$ \\
\hline 12 & $\begin{array}{c}\text { Matriciamento em Saúde Mental: práticas e concepções trazidas por } \\
\text { equipes de referência, matriciadores e gestores }\end{array}$ & Iglesias \& Avellar & 2019 & $\begin{array}{l}\text { Ciência e Saúde } \\
\text { Coletiva }\end{array}$ \\
\hline 13 & $\begin{array}{l}\text { A prática de reuniões de equipes: um dispositivo de cuidado para } \\
\text { trabalhadores de centro de atenção psicossocial (CAPS) }\end{array}$ & Tasca et al. & 2019 & Unoesc \& Ciência \\
\hline 14 & $\begin{array}{l}\text { Apoio matricial como estratégia de ordenação do cuidado em saúde } \\
\text { mental }\end{array}$ & $\begin{array}{c}\text { Lima \& } \\
\text { Gonçalves }\end{array}$ & 2020 & $\begin{array}{l}\text { Trabalho, Saúde e } \\
\text { Educação }\end{array}$ \\
\hline 15 & $\begin{array}{l}\text { Adherence to treatment in collective } \\
\text { multiprofessional activities and factors associated with adherence in } \\
\text { a specialized center for psychosocial care }\end{array}$ & Tergolina et al. & 2020 & $\begin{array}{l}\text { Trends Psychiatry } \\
\text { Psychother }\end{array}$ \\
\hline
\end{tabular}

Fonte: Autores (2021).

No Quadro 2 destacamos os objetivos, tipo de estudo, participantes e instrumento de coleta dos estudos coletados. 
Quadro 2. Distribuição das referências incluídas na revisão integrativa, de acordo com os objetivos, tipo de estudo, participantes e instrumento de coleta.

\begin{tabular}{|c|c|c|c|c|}
\hline $\mathbf{N}^{\mathbf{o}}$ & Objetivo & $\begin{array}{l}\text { Tipo de } \\
\text { estudo }\end{array}$ & Participantes & $\begin{array}{l}\text { Instrumento } \\
\text { de coleta }\end{array}$ \\
\hline 1 & $\begin{array}{c}\text { Compreender experiências de usuários em dois Centros de } \\
\text { Atenção Psicossocial (CAPS), localizados em Fortaleza, Ceará, no } \\
\text { que concerne à qualidade do cuidado conferido pelos } \\
\text { profissionais. }\end{array}$ & Empírico & $\begin{array}{l}\text { Usuários do } \\
\text { CAPS da regional } \\
\text { III e usuários do } \\
\text { CAPS da regional } \\
\mathrm{V}\end{array}$ & $\begin{array}{l}\text { Entrevista não } \\
\text { estruturada e } \\
\text { observação }\end{array}$ \\
\hline 2 & $\begin{array}{l}\text { Caracterizar as produções acerca dos processos de trabalho dos } \\
\text { profissionais dos CAPS, segundo as diretrizes da PNSM. }\end{array}$ & Bibliográfico & Sem participante & $\begin{array}{c}\text { Pesquisa } \\
\text { bibliográfica }\end{array}$ \\
\hline 3 & Discutir as interferências do apoio matricial na atenção à crise. & Empírico & $\begin{array}{l}\text { Profissionais de } \\
\text { um CAPS II }\end{array}$ & $\begin{array}{c}\text { Entrevista } \\
\text { semiestruturada }\end{array}$ \\
\hline 4 & $\begin{array}{c}\text { Explorar o conhecimento e a experiência de uma equipe de Centro } \\
\text { de Atenção Psicossocial Infantil (Capsi) acerca da estratégia do } \\
\text { Apoio Matricial (AM) }\end{array}$ & Empírico & $\begin{array}{l}\text { Profissionais do } \\
\text { CAPS I }\end{array}$ & $\begin{array}{c}\text { Entrevista } \\
\text { semiestruturada }\end{array}$ \\
\hline 5 & $\begin{array}{c}\text { Analisar criticamente a experiência de implantação de um } \\
\text { processo de educação permanente pelos profissionais de um } \\
\text { Centro de Atenção Psicossocial álcool e outras drogas (CAPS ad) } \\
\text { do município de Campinas, SP, Brasil. }\end{array}$ & Empírico & $\begin{array}{l}\text { Profissionais de } \\
\text { um CAPS II }\end{array}$ & $\begin{array}{c}\text { Entrevista } \\
\text { semiestruturada }\end{array}$ \\
\hline 6 & $\begin{array}{l}\text { Caracterizar as práticas e estratégias de Cuidado à Saúde Mental } \\
\text { desenvolvidas pelas equipes de atenção primária na área urbana de } \\
\text { Pesqueira/PE. }\end{array}$ & Empírico & $\begin{array}{l}\text { Profissionais de } \\
\text { um CAPS II }\end{array}$ & $\begin{array}{c}\text { Entrevista } \\
\text { semiestruturada }\end{array}$ \\
\hline 7 & $\begin{array}{c}\text { Conhecer a percepção dos profissionais integrantes da equipe } \\
\text { sobre o trabalho multiprofissional, especialmente no que concerne } \\
\text { aos aspectos que facilitam e dificultam esta atuação. }\end{array}$ & Empírico & $\begin{array}{l}\text { Profissionais de } \\
\text { um CAPS II }\end{array}$ & $\begin{array}{c}\text { Entrevista } \\
\text { semiestruturada }\end{array}$ \\
\hline 8 & $\begin{array}{l}\text { Compreender o cuidado em saúde mental e as articulações com a } \\
\text { rede de atenção à saúde na perspectiva dos profissionais dos } \\
\text { CAPS. }\end{array}$ & Empírico & $\begin{array}{l}\text { Profissionais de } \\
\text { um CAPS I e } \\
\text { CAPS II }\end{array}$ & $\begin{array}{l}\text { Questionário } \\
\text { estruturado/ } \\
\text { Análise do } \\
\text { conteúdo }\end{array}$ \\
\hline 9 & $\begin{array}{l}\text { Analisar como profissionais compreendiam a multi, inter e trans } \\
\text { profissionalidade e como essas práticas aconteciam nos CAPS de } \\
\text { São Paulo/SP. }\end{array}$ & Empírico & $\begin{array}{l}\text { Profissionais de } \\
\text { um CAPS II } \\
\text { e um, CAPS III }\end{array}$ & $\begin{array}{c}\text { Entrevista } \\
\text { semiestruturada }\end{array}$ \\
\hline 10 & $\begin{array}{l}\text { Compreender os desafios enfrentados pelos trabalhadores no } \\
\text { cotidiano da atenção psicossocial, considerando as relações } \\
\text { afetivas, a gestão do cuidado e a interdisciplinaridade. }\end{array}$ & Empírico & $\begin{array}{l}\text { Profissionais } \\
\text { CAPS da regional } \\
\text { IV }\end{array}$ & Narrativas \\
\hline 11 & $\begin{array}{c}\text { compreender a gestão do cuidado na saúde mental sob a } \\
\text { perspectiva da rede de atenção à saúde. }\end{array}$ & Empírico & $\begin{array}{l}\text { Profissionais de } \\
\text { um CAPS }\end{array}$ & $\begin{array}{c}\text { Entrevista } \\
\text { intensiva }\end{array}$ \\
\hline 12 & $\begin{array}{c}\text { Analisar o matriciamento em saúde mental a partir das práticas e } \\
\text { concepções trazidas pelas equipes de referência, equipes } \\
\text { matriciais e gestores a respeito da temática. }\end{array}$ & Empírico & $\begin{array}{l}\text { Profissionais de } \\
\text { um CAPS II }\end{array}$ & $\begin{array}{l}\text { Diário de } \\
\text { campo e análise } \\
\text { de conteúdo }\end{array}$ \\
\hline 13 & $\begin{array}{l}\text { Analisar a perspectiva dos profissionais dos centros de atenção } \\
\text { psicossocial acerca do apoio matricial como estratégia de cuidado } \\
\text { psicossocial em saúde mental. }\end{array}$ & Empírico & $\begin{array}{l}\text { Profissionais de } \\
\text { um CAPS III, III } \\
\text { e IJ }\end{array}$ & $\begin{array}{c}\text { Entrevista } \\
\text { semiestruturada }\end{array}$ \\
\hline 14 & $\begin{array}{l}\text { Este estudo teve como objetivo determinar as taxas de adesão às } \\
\text { atividade multidisciplinares coletivas e identificar os fatores } \\
\text { associados com adesão. }\end{array}$ & Empírico & $\begin{array}{l}\text { Profissionais de } \\
\text { um CAPS }\end{array}$ & $\begin{array}{c}\text { Entrevista } \\
\text { semiestruturada }\end{array}$ \\
\hline 15 & $\begin{array}{l}\text { Compreender as percepções dos profissionais que compõem a } \\
\text { equipe } \\
\text { multidisciplinar de quatro CAPS I, localizados na região Oeste no } \\
\text { Estado de Santa Catarina. }\end{array}$ & Empírico & $\begin{array}{l}\text { Profissionais de } \\
\text { um CAPS I }\end{array}$ & $\begin{array}{l}\text { Entrevista } \\
\text { semiaberta }\end{array}$ \\
\hline
\end{tabular}


Em relação aos objetivos, os estudos voltaram-se para a perspectiva compreender, conhecer, analisar, caracterizar as experiências dos usuários, bem como dos profissionais técnicos de referência. Nos estudos avaliados, identificamos que 14 $(93,33 \%)$ das produções correspondem a estudos empíricos e apenas $01(6,66 \%)$ corresponde a um estudo de cunho bibliográfico. Ao analisarmos os participantes dos estudos, constatamos que 13 (86,66\%) foram os profissionais dos CAPS I, II, III, IV e V; 01 (6,66\%) foram usuários dos CAPS da regional III e usuários do CAPS da regional V e 01 (6,66\%) corresponde a uma pesquisa bibliográfica que não possui participantes. Quanto ao instrumento de coleta, observamos que 08 $(53,33 \%)$ dos estudos utilizaram como instrumento a entrevista semiestruturada, ou seja, segue uma linha pré-definida, mas que se encontra aberto a inclusões; $01(6,66 \%)$ corresponde à entrevista não estruturada e observação; 01 (6,66\%) pesquisa bibliográfica; 01 (6,66\%) questionário estruturado e análise do conteúdo; 01 (6,66\%) narrativas dos profissionais dos CAPS; $01(6,66 \%)$ entrevista intensiva com a equipe multidisciplinar; 01 (6,66\%) diário de campo e análise de conteúdo e 01 (6,66\%) com entrevista semiaberta.

Com a análise dos 15 artigos caracterizamos aspectos acerca das práticas de assistência na rede de atenção saúde mental no país em relação à gestão do cuidado e as equipes multidisciplinares como dispositivos em saúde mental atuantes em CAPS (Quadro 3), sintetizando-as em duas categorias temáticas: aspectos favoráveis e desfavoráveis em relação à gestão do cuidado e equipe multidisciplinar como dispositivos em saúde mental em CAPS.

Na categoria sobre aspectos desfavoráveis em relação à gestão do cuidado e equipe multidisciplinar como dispositivos em saúde mental em CAPS estão a baixa valorização/qualificação 06 (39,96\%), má infraestrutura na continuidade do trabalho $02(13,33 \%)$, isolamento profissional $01(6,66 \%)$, falta de utilização da equipe multidisciplinar em preferência a utilização de medicamento $01(6,66 \%)$, e por fim sobre má gestão do modelo utilizado para tratamento $01(6,66 \%)$. Na categoria dos aspectos favoráveis em relação à gestão do cuidado e equipe multidisciplinar como dispositivos em saúde mental em CAPS surgiu a utilização do apoio matricial como ferramenta apoiadora da atenção à crise $01(6,66 \%)$, utilização de reuniões para troca de experiências 01 (6,66\%), integração da equipe multidisciplinar ao CAPS 01 (6,66\%), por fim, o atendimento humanizador $01(6,66 \%)$.

Quadro 3. Distribuição das subcategorias, categorias e frequência.

\begin{tabular}{|c|c|c|}
\hline Subcategorias & Categorias & Frequência \\
\hline \multirow{5}{*}{$\begin{array}{l}\text { Aspectos desfavoráveis em relação à gestão do } \\
\text { cuidado e equipe multidisciplinar como dispositivos } \\
\text { em saúde mental em CAPS }(73,31 \%)\end{array}$} & Baixa valorização/qualificação & $39,96 \%$ \\
\hline & Má infraestrutura na continuidade do trabalho & $13,33 \%$ \\
\hline & Isolamento profissional & $6,66 \%$ \\
\hline & Má gestão do modelo utilizado para tratamento & $6,66 \%$ \\
\hline & $\begin{array}{l}\text { Falta de utilização da equipe multidisciplinar em } \\
\text { preferência a utilização de medicamentos }\end{array}$ & $6,66 \%$ \\
\hline \multirow{4}{*}{$\begin{array}{c}\text { Aspectos favoráveis em relação à gestão do cuidado e } \\
\text { equipe multidisciplinar como dispositivos em saúde } \\
\text { mental em CAPS }(26,64 \%)\end{array}$} & $\begin{array}{l}\text { Utilização do apoio matricial como ferramenta } \\
\text { apoiadora da atenção à crise }\end{array}$ & $6,66 \%$ \\
\hline & Utilização de reuniões para troca de experiências & $6,66 \%$ \\
\hline & Integração da equipe multidisciplinar ao CAPS & $6,66 \%$ \\
\hline & Atendimento humanizador & $6,66 \%$ \\
\hline
\end{tabular}

Fonte: Autores (2021). 


\section{Discussão}

Nos estudos, as abordagens dos CAPS, se dão na relevância desta modalidade do serviço tem no fortalecimento do modelo de atenção psicossocial e na criação da Rede de Atenção em Saúde Mental, que tem como finalidade oportunizar um cuidado não excludente, de escuta qualificada, possibilitando a reinserção social dos usuários em sofrimento psíquico e maior contato com sua família e comunidade (Sarzana et al., 2018).

\subsection{Aspectos desfavoráveis em relação à gestão do cuidado e equipe multidisciplinar como dispositivos em saúde mental em CAPS}

Na categoria "baixa valorização/qualificação" ( $f=40 \%)$, notou-se a discussão da estrutura física e material do centro acaba por prejudicar o atendimento, assim como a gestão dos medicamentos que os mesmos necessitam em casos mais graves. Neste sentido, o ambiente e relacionamento do profissional, foram considerados precários, além de outras dificuldades ligadas a comunicação da equipe, dificultando assim o trabalho entre os envolvidos no processo do tratamento dos usuários. De modo finalístico os autores notaram que o CAPS presencia uma institucionalização, podendo prejudicar o trabalho dos profissionais, bem como articulação ineficaz com a rede de atenção psicossocial (Pinho et al., 2018).

Além disso, atenta-se aos empecilhos das equipes responsáveis pelos CAPS analisados, que possuem dificuldades comuns, como repasse do cuidado nas reuniões entre as equipes ou em debates organizados nos serviços. Destacamos também a carência na gestão local, abordando atividades que são esquecidas, bem como a ausência ou insuficiência de rotina de reuniões ou oficinas terapêuticas, tal como o mal planejamento da mesma em alguns casos (Borges et al., 2018).

Por meio da literatura coletada, verificou-se que os membros integrantes dos CAPS possuem entendimentos comuns, quando debatido acerca da heterogeneidade da equipe, dificultando a relação e comunicação entre os membros. Reportamos também a dificuldade de manter reuniões para troca de experiências, visto que o cotidiano desses profissionais é intenso e cansativo (Silva \& Knobloch, 2016). Nesta categoria, notamos que a gestão adotada pelos profissionais locais acerca do apoio matricial eram precárias, pois os mesmos possuíam ideias iniciais acerca do tema, porém sendo insuficientes e necessitando de mais debates a respeito para o desenvolvimento e aprimoramento da mesma (Salvador \& Pio, 2016).

Utilizando-se de relatos de profissionais dos CAPS, percebe-se falas direcionadas às dificuldades quanto ao atendimento dos pacientes devido à baixa qualificação, além da sobrecarga ofertada no trabalho interno e a infraestrutura precária para realização do atendimento aos pacientes, reunindo em um mesmo local, pacientes com problemas ou transtornos diferentes. Destaca-se também que, mesmo com a interação dos CAPS com outras instituições de saúde mental, a comunicação entre as mesmas ainda é precária, visto que pacientes que são atendidos não podem desenvolver seu tratamento no local, necessitando ir para outra instituição que pode rejeitar o mesmo, pois não se encaixam na finalidade que o local propõe (Sarzana et al., 2018).

Ainda utilizando de entrevistas com profissionais atuantes em CAPS da cidade de São Paulo, notou-se que algumas respostas às perguntas realizadas possuíam diferentes finais, visto que o entendimento dos profissionais era certeiro e objetivo, enquanto outros acreditavam saber acerca da resposta. Destacou-se, assim, perguntas acerca dos conceitos de "interdisciplinaridade, multidisciplinaridade e transdisciplinaridade", centrando-se na solução de problemas da área, enquanto pontos pertinentes em relação a aspectos comuns foram esquecidos. Além disso, os conceitos de "interprofissional, multiprofissional e transprofissional" também foram perguntados, variando as respostas e aplicação em seus trabalhos. Também se atentou a relatos da dificuldade na aplicação exata das recomendações do Ministério da Saúde acerca do tratamento a saúde mental do paciente, visto que diversas dificuldades dentro do local impedem o atendimento eficiente e recomendado, como sobrecarga no trabalho e sucateamento de equipes, bem como a dificuldade da aplicação dos recursos disponíveis (Jafelice \& Marcolan, 2018). 
Assim como a baixa qualificação dos profissionais acarreta dificuldade no tratamento, a categoria "Má infraestrutura na continuidade do trabalho" $(f=13,33 \%)$ também impede o pleno desenvolvimento do trabalho desses profissionais, evidencia-se que não só a estrutura física, como toda a gestão utilizada para manter o CAPS possui inúmeras dificuldades. Na gestão dos CAPS, destaca-se ainda o crescimento de empresas terceirizadas que disponibilizam profissionais para compor a equipe desses locais (Pessoa et al., 2018).

Destacando pontos ligados a infraestrutura dos CAPS, notou-se que a estrutura física é um fator importante no desenvolvimento, mesmo que seja básica, como a manutenção do local visando o bom atendimento e aplicação do trabalho dos profissionais. Além disso, destacou-se a dificuldade de comunicação com outros locais e instituições para continuidade ou início do tratamento (Lima \& Gonçalves, 2020).

$\mathrm{Na}$ categoria "Isolamento profissional" $(f=6,66 \%)$ destacou-se a dificuldade na comunicação entre as equipes dentro dos CAPS sendo fator determinante para o andamento do atendimento ao paciente, visto que o acordo entre os mesmos devem ser realizados com plena concordância entre os que participam, uma vez que a discrepância ou a negação na realização do pensamento da equipe pode dificultar o processo da melhora do paciente, assim como a dificuldade em realizar reuniões para troca de experiências entre os profissionais atuantes, isolando os mesmos e contendo informações em determinadas pessoas (Filho \& Souza, 2017)

A categoria "Má gestão do modelo utilizado para tratamento" ( $f=6,66 \%)$, visa destacar que ao verificaram a gestão dentro do CAPS, percebe-se que a comunicação entre os profissionais internos dificulta a aplicação da gestão utilizada para tratamento dos pacientes, devido a precarização da qualificação, assim como o isolamento de outros profissionais, visto que a recusa no debate de assuntos já discutidos e tidos como "desnecessários" faz com que a exploração mais profunda dos temas seja vista como "inútil" (Iglesias \& Avellar, 2019).

$\mathrm{Na}$ categoriza "Falta de utilização da equipe multidisciplinar em preferência a utilização de medicamentos" $(f=6,66 \%)$, utilizando o modelo de atividades multiprofissionais coletivas (CMPA), notou-se que a falta de utilização das equipes multidisciplinares dentro dos CAPS por motivos como a infraestrutura, inadequação dos profissionais e reluta dos pacientes, direcionam os pacientes, por desejarem um resultado mais rápido, a optarem por tratamentos com utilização de medicamentos mais agressivos, visando atenuar ou conter a enfermidade mental que enfrentam (Tergolina et al., 2020).

\subsection{Aspectos favoráveis em relação à gestão do cuidado e equipe multidisciplinar como dispositivos em saúde mental em CAPS}

A categoria "utilização do apoio matricial como ferramenta apoiadora da atenção à crise" ( $f=6,66 \%)$ vem na perspectiva de discutir as questões das ações do matriciamento a atenção à crise no que diz respeito a atenção psicossocial nos CAPS (Lima \& Dimenstein, 2016). Com isso, podemos constatar que o apoio matricial e equipe de referência são arranjos organizacionais, bem como uma metodologia para a gestão do trabalho em saúde.

As ações estratégicas da Política Nacional de Saúde Mental (PNSM) pontua que os CAPS são serviços que ocupam função estratégica na missão de substituição da lógica manicomial (Lima \& Dimenstein, 2016). Ou seja, configuram-se como ações estratégicas da PNSM a serem fortalecidas e por estarem, no contexto atual da micropolítica do trabalho, na ordem de prioridade de questões e de inquietações dos trabalhadores.

A equipe multidisciplinar como dispositivos técnicos de referência em saúde mental nos CAPS propõe um novo sistema de referência entre profissionais e usuários, cujo funcionamento pode ser descrito da seguinte forma: cada unidade de saúde se organiza por meio da composição de equipes, formadas segundo características e objetivos da própria unidade, e de acordo com a realidade local e disponibilidade de recursos (Lima \& Dimenstein, 2016). 
Essas equipes obedecem a uma composição multiprofissional de caráter transdisciplinar, isto é, reúnem profissionais de diferentes áreas, variando em função da finalidade do serviço/unidade. Cada equipe de referência torna-se responsável pela atenção integral do paciente, cuidando de todos os aspectos de sua saúde, elaborando projetos terapêuticos e buscando outros recursos terapêuticos, quando necessário. Com isso, vem se destacando um conjunto de estratégias e recursos, os quais são utilizados pela equipe de referência dos CAPS utilizados no cuidado à crise, como: horário estendido; acolhimento; encaminhamento; intervenção medicamentosa; contenção física; cuidado intensivo; reinserção e reabilitação psicossocial; assistência domiciliar e familiar; ações territoriais (Lima \& Dimenstein, 2016).

Em relação ao cuidado à crise, o apoio matricial visa a relevância para a construção de uma Atenção Psicossocial à crise. Dessa forma, é um instrumento imprescindível para garantir o desenvolvimento das ações integrais à saúde, principalmente a saúde pública.

A categoria "utilização de reuniões para troca de experiências" ( $f=6,66 \%)$, o matricialmente pode ser entendido como arranjo organizacional que visa responder o princípio da integralidade, e, em relação à prática, amplia e qualifica o escopo de ações das equipes da Atenção Básica (Lima \& Dimenstein, 2016).

As reuniões feitas pelas equipes multidisciplinares em saúde mental são consideradas essenciais para o desenvolvimento da equipe tal como de seu trabalho no CAPS, pois possibilitam que a equipe de referência reflita suas práticas e o gerenciamento do cuidado no ambiente em que atua. As reuniões das equipes matriciais atuantes em CAPS auxilia tanto na organização do trabalho e estruturação da unidade, quanto para proporcionar um momento de cuidado (Tasca \& Biesdorf, 2019). É imprescindível para a saúde tanto do paciente/usuário quanto para os colegas de trabalho, mostrando a importância da socialização e do que é feito entre os profissionais, tanto para o bom funcionamento dos CAPS quanto para o bem-estar dos profissionais.

É nesta perspectiva que se destaca a categoria "integração da equipe multidisciplinar ao CAPS" ( $f=6,66 \%)$, esse movimento vem com a finalidade de desenvolver as possibilidades terapêuticas, resolvendo o risco de fragmentação do trabalho, na perspectiva de impor a necessidade de integração verdadeiramente interdisciplinar. É importante destacar que a partir da década de 1960, a proposta interdisciplinar ganha força, na perspectiva de superação da crescente fragmentação do conhecimento. A interdisciplinaridade versa sobre a intensidade das trocas entre os especialistas e sobre o grau de integração das disciplinas em um projeto profissional, de ensino ou de pesquisa (Barbosa et al., 2017). Ou seja, envolve como estratégia a troca real de conhecimentos e uma integração mais profunda e coordenada entre disciplinas, diferente da multidisciplinaridade, essa limitada à simples justaposição de várias disciplinas em função da realização de determinado trabalho.

Diante disto, a integração das equipes multidisciplinares como técnicos de referência foca-se na troca de saberes entre equipes, construindo forças para ampliar as ações e modificar os problemas de saúde, promovendo a ampliação nas ações de saúde (Lima \& Dimenstein, 2016).

E por fim, a última categoria "atendimento humanizador" $(6,66 \%)$ é necessário como meio de resgate ao respeito no atendimento ao usuário, assim, é importante que prestar cuidado humanizado seja uma finalidade de toda a equipe multidisciplinar. Assim, destacamos o acolhimento, o trabalho de educação em saúde e o cuidado humanizado nos CAPS garantem uma relação baseada no diálogo entre os profissionais e usuários, portanto, são fundamentais para uma assistência de qualidade para os usuários (Andrade \& Bosi, 2015).

\section{Considerações Finais}

A partir da presente revisão integrativa observou-se que o CAPS é considerado uma instituição que se encontra em constante melhoria, além de seu importante papel na saúde pública, cuja sua prestação de serviço é voltada aos usuários que se encontram em sofrimento psíquico. Para isso, foram estudados os aspectos desfavoráveis em relação à gestão do cuidado e 
equipe multidisciplinar como dispositivos em saúde mental dentre eles destacamos: a baixa valorização/qualificação profissional; infraestrutura precária, má gestão e a dificuldade na comunicação entre as equipes a qual é fator determinante para o andamento do atendimento ao paciente. Com relação aos aspectos favoráveis podemos ressaltar: a utilização do apoio matricial como ferramenta apoiadora da atenção à crise, a utilização de reuniões para troca de experiências e a integração da equipe multidisciplinar.

Entretanto, cabe destacar que o atendimento humanizado deve ser focado no acolhimento, no trabalho de educação em saúde e na gestão do cuidado baseada no diálogo entre os profissionais e os usuários. Por isso, pontuamos que são necessários mais estudos que abordam as formações oferecidas pelos CAPS, para que o profissional possa, diante de instruções complementares, desempenhar melhores resultados diante de seu trabalho, para com a melhora dos clientes e difusão de experiências positivas ou negativas obtidas com os mesmos.

Portanto, concluímos enfatizando sobre o importante papel da equipe multidisciplinar em saúde mental, técnicos de referência, profissionais responsáveis pelo monitoramento do projeto terapêutico individual, contato com a família dos usuários, assim como pela avaliação das metas traçadas em relação ao serviço prestado ao usuário.

\section{Referências}

Andrade, A. B., \& Bosi, M. L. M. (2015). Qualidade do cuidado em dois centros de atenção psicossocial sob o olhar de usuários. Saude e Sociedade, 24(3), 887-900. https://doi.org/10.1590/S0104-12902015131949

Barbosa, V. F., Cavalcanti, A., Alcântara, M. C. D. A., Pedroza, R. D. M., \& Ferreira, S. H. V. (2017). O papel da atenção primária de saúde na constituição das redes de cuidado em saúde mental The role of primary attention in health on the constitution of the network care in mental health. Revista de Pesquisa: Cuidado é Fundamental Online, 9(3), 659. https://doi.org/10.9789/2175-5361.2017.v9i3.659-668

Borges, K. C. S. S., Rodrigues, J. M., Gonçalves, L. L. M., Souza, P. C. da S., Souza, T. de P., \& Lamy, Z. C. (2018). O Cuidado nos CAPS numa Região de Saúde Maranhense. Rev. Polis e Psique, 8(1), 92-111.

Brasil. (2002). Portaria GM n $n^{\circ}$ 336/GM de 19 de fevereiro de 2002. Define e estabelece sobre as modalidades de serviços dos Centros de Atenção Psicossocial. Brasília, DF: Ministério da Saúde. http://portal.saude.gov.br/portal/arquivos/pdf/Portaria GM 336-2002.pdf

Brasil. (2004). HumanizaSUS: equipe de referência e apoio matricial/Ministério da saúde. Núcleo Técnico da Política Nacional de Humanização. Brasília: Ministério da Saúde.

Filho, N. C. A., \& Souza, A. M. P. de. (2017). A percepção sobre o trabalho em equipe multiprofissional dos trabalhadores de um Centro de Atenção Psicossocial em Salvador, Bahia, Brasil. Interface: Communication, Health, Education, 21(60), 63-76. https://doi.org/10.1590/1807-57622015.0428

Ganong, L. H. (1987). Integrative reviews of nursing research. Research in Nursing \& Health, 10(1), 11.

Iglesias, A., \& Avellar, L. Z. (2019). Matrix support in mental health: Practices and concepts brought by reference teams, matrix teams and managers. Ciencia e Saude Coletiva, 24(4), 1247-1254. https://doi.org/10.1590/1413-81232018244.05362017

Jafelice, G. T., \& Marcolan, J. F. (2018). O trabalho multiprofissional nos Centros de Atenção Psicossocial de São Paulo. Rev. Bras. Enferm, 71(supl.5), 21312138. http://www.scielo.br/scielo.php?script=sci_arttext\&pid=S0034-71672018001102131

Lima, M. C., \& Gonçalves, T. R. (2020). Apoio Matricial Como Estratégia De Ordenação Do Cuidado Em Saúde Mental. Trabalho, Educação e Saúde, 18(1), 1-21. https://doi.org/10.1590/1981-7746-sol00232

Lima, M., \& Dimenstein, M. (2016). O apoio matricial em saúde mental: Uma ferramenta apoiadora da atenção à crise. Interface: Communication, Health, Education, 20(58), 625-635. https://doi.org/10.1590/1807-57622015.0389

Mendes, K. D. S., Silveira, R. C. de C. P., \& Galvão, C. M. (2008). Revisão integrativa: método de pesquisa para a incorporação de evidências na saúde e na enfermagem. Texto \& Contexto - Enfermagem, 17(4), 758-764. https://doi.org/10.1590/s0104-07072008000400018

Pessoa, K. L. V., Jorge, M. S. B., Lourinho, L. A., \& Catrib, A. M. F. (2018). Gestão do cuidado e interdisciplinaridade: desafios do cotidiano da atenção psicossocial. Revista de Salud Pública, 20(6), 692-698. https://doi.org/10.15446/rsap.v20n6.64641

Pinho, E. S., Souza, A. C. S., \& Esperidião, E. (2018). Processos de trabalho dos profissionais dos centros de atenção psicossocial: Revisão integrativa. Ciencia e Saude Coletiva, 23(1), 141-152. https://doi.org/10.1590/1413-81232018231.08332015

Salvador, D. B., \& Pio, D. A. M. (2016). Apoio Matricial e Capsi: desafios do cenário na implantação do matriciamento em saúde mental. Saúde Em Debate, 40(111), 246-256. https://doi.org/10.1590/0103-1104201611119

Sarzana, M. B. G., Lessa, G., Preis, L. C., Perin, J. P. da L., Andrade, S. R. de, \& Erdmann, A. L. (2018). Mental Health Care Management From the Perspective of the Health Care Network. REME: Revista Mineira de Enfermagem, 22, 1-8. https://doi.org/10.5935/1415-2762.20180075 
Research, Society and Development, v. 10, n. 6, e21010615747, 2021

(CC BY 4.0) | ISSN 2525-3409 | DOI: http://dx.doi.org/10.33448/rsd-v10i6.15747

Silva, D. L. S., \& Knobloch, F. (2016). A equipe enquanto lugar de formação:A educação permanente em um Centro de Atenção Psicossocial álcool e outras drogas. Interface: Communication, Health, Education, 20(57), 325-335. https://doi.org/10.1590/1807-57622015.0061

Tasca, P. C., \& Biesdorf, A. (2019). Um Dispositivo De Cuidado Para Trabalhadores De Centros De Atenção Psicossocial ( Caps ). Unoesc \& Ciência, 10(2), 99-106.

Tergolina, L. P., Stein, A. T., \& de Faria, E. R. (2020). Adherence to treatment in collective multiprofessional activities and factors associated with adherence in a specialized center for psychosocial care. Trends in Psychiatry and Psychotherapy, 42(4), 329-339. https://doi.org/10.1590/2237-6089-2019-0053

Whittemore, R., \& Knaf, K. (2005). The integrative review: updated methodology Robin. METHODOLOGICAL ISSUES IN NURSING RESEARCH, 52(5), 546-553. https://doi.org/10.1016/j.pmn.2007.11.006 